\title{
A Rare Case of Unilateral Pleural Effusion in a Pediatric Patient on Chronic Peritoneal Dialysis: Is it a Pleuroperi- toneal Leakage?
}

\author{
Sukdong Yoo, M.D.' \\ Jae-Yeon Hwang, M.D., Ph.D. ${ }^{2}$ \\ JiYeon Song, M.D. ${ }^{1}$ \\ Taek Jin Lim, M.D. ${ }^{1}$ \\ Narae Lee, M.D. ${ }^{1}$ \\ Su Young Kim, M.D., Ph.D. ${ }^{1}$ \\ Seong Heon Kim, M.D ${ }^{1}$ \\ Department of Pediatrics ${ }^{1}$, Pusan \\ National University Children's Hospital, \\ Korea, Department of Radiology ${ }^{2}$, \\ Pusan National University Children's \\ Hospital, Korea
}

\section{Corresponding author: \\ Seong Heon Kim, M.D. \\ Department of Pediatrics, Pusan National University Children's Hospital, 20 Geumo- ro, Mulgeum-eup, Yangsan, Goungnam \\ 50612, Korea \\ Tel: +82-55-360-3163 \\ Fax: +82-55-360-2181 \\ E-mail:pedksh@gmail.com}

Received: 15 September 2018

Revised: 4 October 2018

Accepted: 10 October 2018
Non-infectious complications of peritoneal dialysis (PD) are relatively less common than infectious complications but are a potentially serious problem in patients on chronic PD. Here, we present a case of a non-infectious complication of PD in a 13year- old boy on chronic PD who presented with symptoms such as hypertension, edema, dyspnea, and decreased ultrafiltration. Chest and abdominal radiography showed pleural effusion and migration of the PD catheter tip. Laparoscopic PD catheter reposition was performed because PD catheter malfunction was suspected. However, pleural effusion relapsed whenever the dialysate volume increased. To identify peritoneal leakage, computed tomography (CT) peritoneography was performed, and a defect of the peritoneum in the left lower abdomen with contrast leakage to the left rectus and abdominis muscles was observed. He was treated conservatively by transiently decreasing the volume of night intermittent PD and gradually increasing the volume. At the 2-year follow-up visit, the patient had not experienced similar symptoms. Patients on PD who present with refractory or recurrent pleural effusion that does not respond to therapy should be assessed for the presence of infection, catheter malfunction, and pleuroperitoneal communication. Thoracentesis and CT peritoneography are useful for evaluating pleural effusion, and timely examination is important for identifying the defect or fistula.

Key words: Peritoneal dialysis, Pleuroperitoneal communication, Peritoneography, Pleural effusion, PD catheter

\section{Introduction}

Noninfectious complications of peritoneal dialysis (PD) are relatively less common than infectious complications, but are a potentially serious problem in patients on chronic PD. The well-known complications include catheterrelated complications, such as leakage, hernia, hydrothorax, hemoperitoneum, pancreatitis, ischemic colitis, necrotizing enterocolitis, pneumoperitoneum, and metabolic complications ${ }^{1,2}$.

Occasionally, such complications may have resulted from a combination of one or more causes, or they can be confused as a different condition. Thus, a systematic approach is needed when identifying the causes of the PD complications. We present herein a patient on chronic PD who presented with unilateral pleural effusion. 


\section{Case report}

A 13-year-old boy on chronic PD was admitted due to weight gain, edema, and hypertension. He was diagnosed with chronic kidney disease (CKD) secondary to focal segmental glomerulosclerosis due to reflux nephropathy 4 years prior to his admission. Although he remained polyuric, his uremia was getting worse. Thus, he underwent PD 4 months prior. At that time, night intermittent PD (NIPD) consisting of 5 cycles of 1.5 liter exchanges with $1.5 \%$ dextrose-based solution for 10 hours was performed. He does not have a history of heart disease, peritonitis, hernia, and surgery of the thorax or abdomen.

While playing, this very active boy was kicked by his friend on his abdomen 10 days prior to his admission. Following the incident, he complained of intermittent left upper quadrant (LUQ) pain. He initially presented with a $5.6 \mathrm{~kg}(10.8 \%)$ body weight gain, hypertension, and generalized edema with decreased ultrafiltration. Antihypertensive drugs, such as losartan and amlodipine, and diuretics, such as furosemide, were prescribed in the outpatient clinic, but symptoms still progressed. Subsequently, he pre-

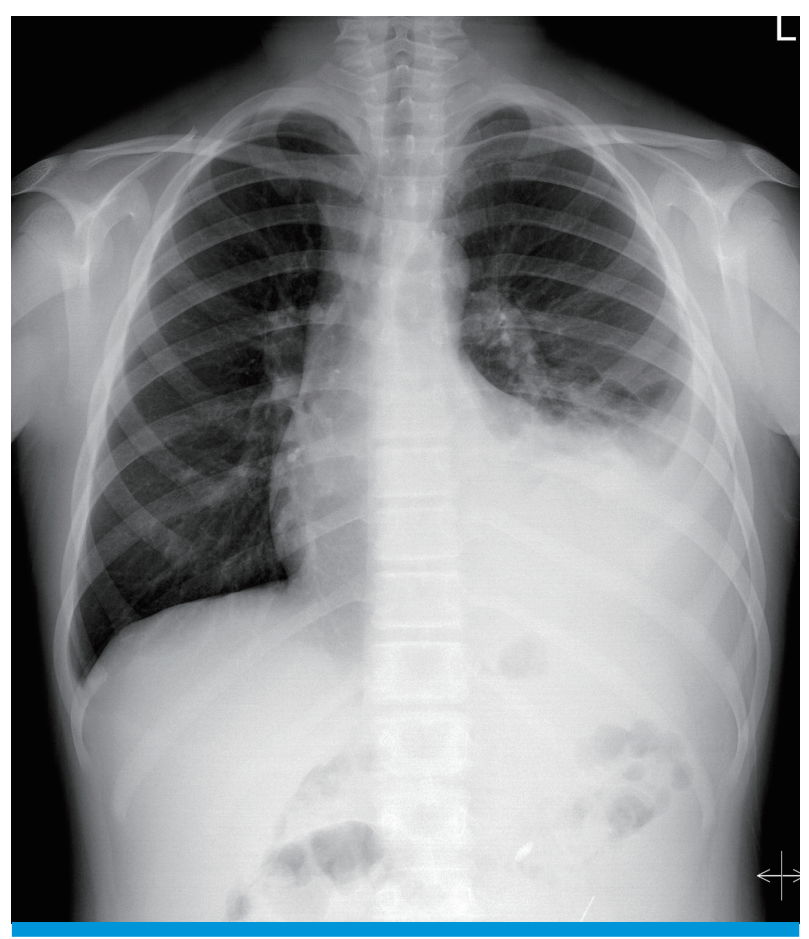

Fig. 1. Initial chest postero-anterior view. There is a large amount of left pleural effusion with passive atelectasis of the left lower lung field. Note that pulmonary vascularity of both lungs is normal. sented to the emergency room with dyspnea and hypertension, and was then admitted. Initial vital signs were as follows: respiration rate, 25 breaths/min; heart rate, 105 beats/ min; and blood pressure, $180 / 100 \mathrm{mmHg}$.

Laboratory findings, including serum albumin level, were normal except for blood urea nitrogen and creatinine levels. Chest and abdominal radiography showed left-sided pleural effusion (Fig. 1) and PD catheter tip migration to the LUQ (Fig. 2). We assumed that hypertension and pleural effusion may have occurred due to ultrafiltration failure caused by the malfunctioning catheter, which moved to an inadequate position.

PD was stopped transiently. Laparoscopic peritoneal dialysis catheter repositioning was done without complications. Operative findings showed that the catheter tip was located and attached in front of the left colon. Omental wrapping and intraluminal fibrin formation were absent. Catheter function test was performed, revealing optimal catheter function.

Initially, we planned to temporally discontinue PD to allow healing; however, renal failure symptoms, such as electrolyte imbalance, immediately occurred, and the patient and caregiver strongly refused hemodialysis. At postoperative day (POD) 1, continuous ambulatory PD (CAPD)

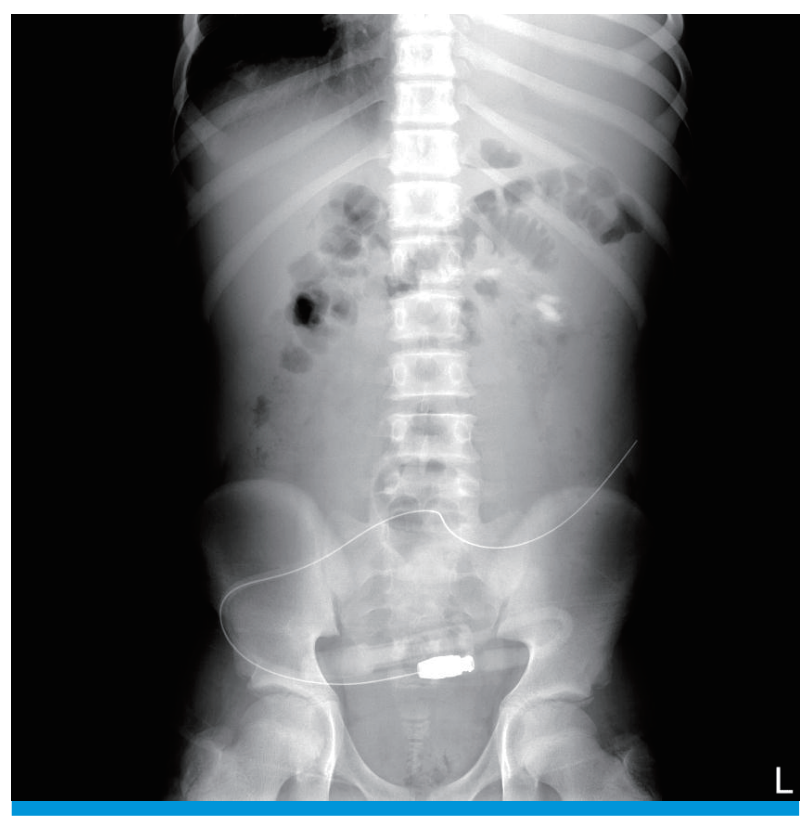

Fig. 2. Initial abdomen postero-anterior view. There is migration of peritoneal dialysis catheter tip to left upper quadrant, which was initially located in the pelvic cavity. Note that left pleural effusion is present. 
was started using $500 \mathrm{~mL}$ every 6 hours. At POD 2, the dialysate volume was increased to $750 \mathrm{~mL}$ every 6 hours. During dialysis, no leakage occurred, and ultrafiltration was fair. At POD 3, he had a mild cough and decreased lung sound at the left lower lobe. Chest radiography showed mild left-sided pleural effusion. The dialysate volume was the reduced to $500 \mathrm{cc}$. On the next day, follow-up chest radiography showed improvement of pleural effusion. Although repositioning of the catheter was performed, pleural effusion exacerbated repeatedly whenever the dialysate volume was increased. Because we suspected the presence of peritoneal leakage into the pleural space, CT peritoneography following the method of Cochran et al. was performed. After the peritoneal cavity was completely drained of the dialysate, nonionic contrast material was mixed with the dialysate and infused into the peritoneal cavity, and the patient was encouraged to walk for 1 hour until undergoing CT to achieve good distribution. CT peritoneography showed a defect of the peritoneum in the left lower abdomen with a contrast leakage to the left rectus and abdominis muscles (Fig. 3) ${ }^{3}$. The patient was treated conservatively by decreasing the NIPD volume, and then slowly increasing the volume again. The initial regimen of the NIPD was 7 cycles of $500 \mathrm{~mL}$ exchanges for 10 hours with $4.25 \%$ dextrose solution, and the dialysate volume was gradually increased to 1.5 liter within 2 months. The conservative treatment performed successfully managed the patient's condition. For the next two years, the patient had not encountered similar problems during his outpatient clinic follow-up visits.

\section{Discussion}

Pleural effusion in patients with CKD is uncommon but is a well-recognized complication. In children, the incidence of pleural effusion ranges from $0.66 \%$ to $3 \%$, . It has a diverse etiology, such as heart failure, tuberculosis, uremic effusion, and volume overload. In patients on PD, pleural effusion is typically managed by increasing the dialysate

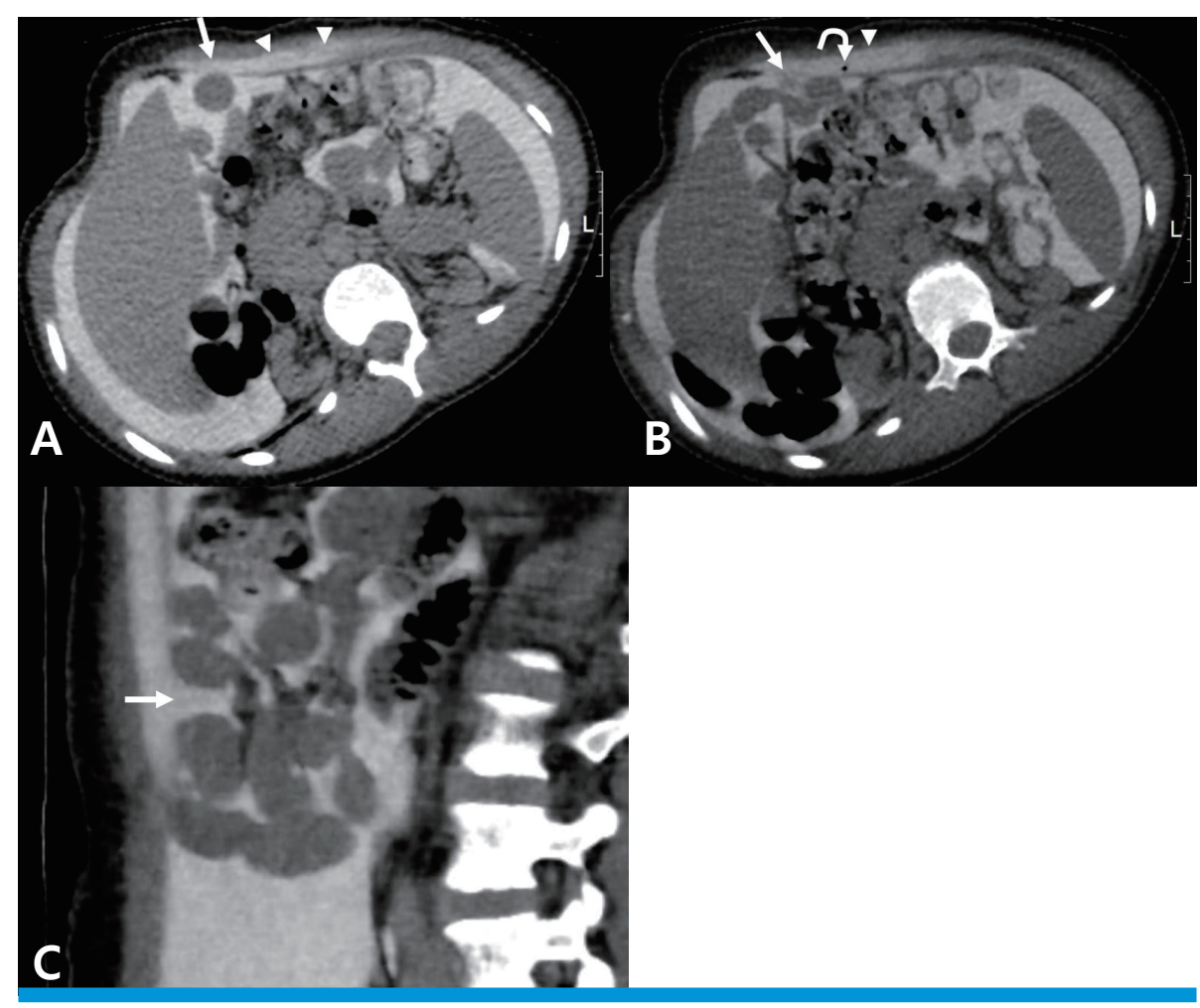

Fig. 3. Computed tomography peritoneography after 1 hour of injecting a water-soluble iodinated contrast media in an oblique prone position. Axial images obtained at the L1 (A) and L3 (B) level and a sagittal reconstructed image (C) show focal defect of the peritoneum (arrows). Note the contrast leakage (arrowheads) to the abdominal wall with tiny air bubble (curved arrow). 
volume and ultrafiltration. However, if pleural effusion remained as a refractory problem despite proper medical treatment, the presence of infection, catheter-related complications, and pleuroperitoneal communication (PPC) should be considered ${ }^{6,7)}$.

Unfortunately, PPC is a putative diagnosis in this case because the findings during the operation and on CT peritoneography were not sufficient to reveal a direct clue about the route between PPC and the defect of the peritoneum. However, the relationship between PPC and the defect of the peritoneum is possible for the following reasons. First, the environment, such as increased intraperitoneal pressure, can cause a defect of the peritoneum and PPC. Second, both pleural effusion and peritoneal defect occurred ipsilaterally. Third, PPC is known to be rare on the left, but only left-sided pleural effusion was identified whenever the volume of the dialysate was increased.

European Paediatric Dialysis Working Group reported the following risk factors of PPC: young age, hernia, abdominal surgery, and peritonitis ${ }^{5}$. Suggested risk factors of PD catheter migration from a retrospective review are constipation, greater omentum wrapping, and operation technique, such as catheter insertion site and catheter type ${ }^{8)}$.

In our case, there was no risk factor of PPC, catheter migration, and peritoneal defect. Location of the defect was at the left lower quadrant; thus, it was not related to PD catheter insertion, which was performed at the right lower quadrant. Left-sided pleural effusion, which was ipsilateral with the peritoneal defect, always recurred whenever increasing the dialysate volume. A large multicenter study in Japan reported that $88 \%$ of pleural effusion cases was in the right side in PPC. On the other hand, the incidence of left pleural effusion is lower because the pericardium covers the defect and prevents peritoneal fluid leakage into the left pleural space ${ }^{9}$. These findings suggest that left-sided pleural effusion may be related to left peritoneal defect.

Although we did not perform diagnostic thoracentesis because spontaneous resolution was quickly achieved, thoracentesis and pleural fluid analysis was useful in revealing transudate and high pleural fluid-to-serum glucose ratio confirming the clinical suspicion of PPC. However, it is important to note that the initial pleural fluid can be exudates and can have low pleural fluid-to-serum glucose $\operatorname{gradient}^{10)}$.
CT peritoneography was performed, which revealed the defect of the peritoneum. However, we could not identify direct evidence of PPC, which could be due to the limitation or timing of the test, because CT was performed after the disappearance of pleural effusion.

We hypothesized that abdominal trauma or increased pressure in abdominal cavity may cause peritoneal defect and PPC, resulting in pleural effusion ${ }^{11}$. There was minor abdominal trauma history of the patient in our case. However, atraumatic peritoneal damage or intrinsic defect cannot be ruled out. There were some cases of atraumatic damage of the intraabdominal organ, such as spleen rupture, bowel erosion, and perforation. Retrospective studies of the serial changes of CT peritoneography in patients with symptomatic ultrafiltration failure have shown that retroperitoneal leakage, anterior abdominal wall leakage, and inguinal hernia can occur without trauma ${ }^{12-14)}$. Intra-abdominal pressure in patients on PD can be changed depending on the patients' position, dialysate volume, and coughing. Moreover, the incidence of hernia and peritoneal leaks are higher in the PD population. However, whether increasing the hydrostatic intraabdominal pressure increases the risk of abdominal wall complications still remains controversial. In this regard, some authors insisted that intrinsic peritoneal defect may have existed prior to the presentation of complication ${ }^{15)}$.

In conclusion, in patients on PD who present with refractory or recurrent pleural effusion that does not respond to therapy, including increasing the dialysate volume or concentration for volume overloading, another factor such as infection, catheter malfunction, and PPC should be considered. To evaluate pleural effusion, thoracentesis and CT peritoneography are useful, but timely appropriate examination is important to identify the defect or fistula.

\section{Patient consent}

This study was approved by the institutional review board (IRB), and the consent was waived due to the nature of the retrospective study [IRB number 05-2018-155]. 


\section{Conflicts of interest}

\section{No potential conflict of interest relevant to this article was reported.}

\section{References}

1. Kim JE, Park SJ, Oh JY, Kim JH, Lee JS, Kim PK, et al. Noninfectious Complications of Peritoneal Dialysis in Korean Children: A 26-Year Single-Center Study. Yonsei Med J 2015;56:1359-64.

2. Saha TC, Singh H. Noninfectious complications of peritoneal dialysis. South Med J 2007;100:54-8.

3. Cochran ST, Do HM, Ronaghi A, Nissenson AR, Kadell BM. Complications of peritoneal dialysis: evaluation with CT peritoneography. Radiographics 1997;17:869-78.

4. Kawaguchi AL, Dunn JC, Fonkalsrud EW. Management of peritoneal dialysis-induced hydrothorax in children. Am Surg 1996;62: 820-4.

5. Dufek S, Holtta T, Fischbach M, Ariceta G, Jankauskiene A, Cerkauskiene $\mathrm{R}$, et al. Pleuro-peritoneal or pericardio-peritoneal leak in children on chronic peritoneal dialysis-A survey from the European Paediatric Dialysis Working Group. Pediatr Nephrol 2015; 30:2021-7.

6. Lew SQ. Hydrothorax: pleural effusion associated with peritoneal dialysis. Perit Dial Int 2010;30:13-8.

7. Ray S, Mukherjee S, Ganguly J, Abhishek K, Mitras S, Kundu S. A cross-sectional prospective study of pleural effusion among cases of chronic kidney disease. Indian J Chest Dis Allied Sci 2013; 55:209-13.

8. Lan L, Jiang J, Wang P, Ren W, Hu Z. Peritoneal dialysis catheter placement in the right lower quadrant is associated with a lower risk of catheter tip migration: a retrospective single-center study. Int Urol Nephrol 2015;47:557-62.

9. Nomoto Y, Suga T, Nakajima K, Sakai H, Osawa G, Ota K, et al. Acute hydrothorax in continuous ambulatory peritoneal dialysis--a collaborative study of 161 centers. Am J Nephrol 1989;9:363-7.

10. Momenin N, Colletti PM, Kaptein EM. Low pleural fluid-to-serum glucose gradient indicates pleuroperitoneal communication in peritoneal dialysis patients: presentation of two cases and a review of the literature. Nephrol Dial Transplant 2012;27:1212-9.

11. Mahale AS, Katyal A, Khanna R. Complications of peritoneal dialysis related to increased intra-abdominal pressure. Adv Perit Dial 2003;19:130-5.

12. Cheung M, Chu FS, Kwan LP. Serial changes of computed tomographic peritoneogram in patients with symptomatic ultrafiltration failure complicating continuous ambulatory peritoneal dialysis. J Med Imaging Radiat Oncol 2017;61:321-6.

13. Fujiwara M, Soda T, Okada T, Kanamaru H, Inoue T, Ogawa O. Bowel perforation by a peritoneal dialysis catheter: report of two cases. BMC Nephrol 2017;18:312.

14. Kinoshita C, Quy PN, Honda M. Atraumatic splenic rupture in a peritoneal dialysis patient. CEN case reports. 2018.

15. Del Peso G, Bajo MA, Costero O, Hevia C, Gil F, Diaz C, et al. Risk factors for abdominal wall complications in peritoneal dialysis patients. Perit Dial Int 2003;23:249-54. 